\title{
Similarity Measure for Corner Redetection
}

\author{
Christoph Stock and Axel Pinz \\ Institute of Electrical Measurement and Measurement Signal Processing \\ Graz University of Technology, Austria \\ \{stock, pinz\}@emt.tugraz.at \\ http://www.emt.tugraz.at/ tracking
}

\begin{abstract}
Corners are important image-features for tracking applications. We present a new method to calculate the similarity of corners, which is used to improve the redetection performance of corner-based tracking applications. It is a simple and fast method to calculate a scaled measure of similarity, which aggregates basic corner features like dihedral angle, cornerness, and corner orientation. Experimental results verify that the similarity measure is well suited for tracking applications.
\end{abstract}

\section{Introduction}

Corners are important features for image analysis. Their detection is often required in many computer vision applications, like object recognition, pattern matching or motion tracking.

Motivated by the weak performance of corner-based tracking applications which are using small subwindows, we develop a measure of similarity for corners to improve the robustness of our tracking application, which was presented in [1]. Our model-based method is very sensitive to rotation changes, but in contrast to typical matching methods [9], like correlation based methods, robust against lighting variations, because our method doesn't work on the pure intensity values. This behavior meets our requirements for tracking applications very well, because the corner orientation varies only little between consecutive frames and on the other hand lighting conditions can rapidly change depending on the light source (e.g. fluorescent tubes).

Besides their position, corners contain additional information, like dihedral angle, corner orientation and a measure of cornerness. Our approach uses this additional information for a precise description of the detected corner. With the help of this description we are able to develop a corner similarity measure. This similarity measure can be used for redetection of known corners or as a quality measure for tracking applications which use corners for motion reconstruction. This yields more robust tracking applications.

The main contribution of this paper is high redetection performance paired with low computational cost, so we are able to use this algorithm for real-time tracking applications [5]. 


\section{$1.1 \quad$ Related Work}

Several papers try to parameterize corners for matching purpose and describe corners within a local neighborhood [8],[3]. Others use correlation techniques and work directly on the intensity values [9], and therefore lack robustness against lighting variations. Others work by calculating the Hausdorff distance [4].

\section{Similarity Measure}

A detected corner given by a certain pre-procedure, like Harris[2], Stock[10] or Rohr[6], can be described by several corner-features $F^{i}$. We compare a corner in a reference image with features $F_{r e f}^{i}$ with a corner in a test image with features $F_{\text {test }}^{i}$ (see eq. 1). By analyzing these Similarity Criteria $S C_{i}$ we are able to formulate a measure of similarity $S M$ (see eq. 2):

$$
\begin{aligned}
& S C_{i}=f\left(F_{r e f}^{i}, F_{\text {test }}^{i}\right) \geq 0 \quad \text { eg. } S C_{i}=\left|F_{\text {ref }}^{i}-F_{\text {test }}^{i}\right|, \\
& S M=\frac{\sum_{i} a_{i} e^{-\frac{S C_{i}}{2 \pi \sigma}}}{\sum_{i} a_{i}} \quad 0<S M \leq 1 .
\end{aligned}
$$

One can use the parameter $a_{i} \in[0 \ldots 1]$ to weight the different Similarity Criteria $S C_{i}$. By using values other than 0 or 1 for $a_{i}$, one is able to modify the impact for different criteria. Additionally it is possible to modify the sensitivity of each Similarity Criterion $S C_{i}$ by changing $\sigma$ of the exponential part of equation 2. This is also useful for different applications. A Similarity Measure $S M=1$ indicates an identical corner, on the other side a value of 0 for the measure of similarity $S M$ marks very dissimilar corners.

Depending on the application and on the underlying corner detector, different criteria like cornerness $(C N)$, corner-orientation $(\alpha)$, dihedral angle $(\beta)$ and $\mathrm{x}$ and y-displacement $(X$ and $Y$ ) can be used. Subsection 2.1 gives a brief overview on how to calculate the dominant gradient directions of a corner, which are used to calculate the orientation and the dihedral angle of a detected corner.

For a typical tracking application which should deliver the best fit corner of the next frame, the Similarity Measure $S M$ can be defined as follows:

$$
\begin{gathered}
S M=\frac{1}{\sum_{i=0}^{4} a_{i}}\left(a_{0} e^{-\frac{\left|X_{r e f}-X_{t e s t}\right|}{2 \pi \sigma}}+a_{1} e^{-\frac{\left|Y_{r e f}-Y_{t e s t}\right|}{2 \pi \sigma}}+a_{2} e^{-\frac{\left|\alpha_{r e f}-\alpha_{t e s t}\right|}{2 \pi \sigma}}+\right. \\
\left.a_{3} e^{-\frac{\left|\beta_{r e f}-\beta_{t e s t}\right|}{2 \pi \sigma}}+a_{4} e^{-\frac{\left|C N_{r e f}-C N_{t e s t}\right|}{2 \pi \sigma}}\right) .
\end{gathered}
$$

It is obvious that $a_{0}$ and $a_{1}$ should be zero for normal corner redetection within 
the whole image. Only for tracking applications where the position of a corner varies only a little between subsequent frames, it is useful to consider this information.

In contrast to other methods on calculating a similarity measure, like correlationbased methods [9] or methods based on calculating the Hausdorff distance [4], our model-based method is a simple and fast alternative solution, ideally suited for corner-redetection in tracking applications. Without using an explicit metric, we achieve a scaled measure of similarity $(S M \in[0,1])$.

\subsection{Dominant Gradient Directions}

Due to the fact that corners are intersections of two or more edges, we are able to parameterize the corner by the description of the involved edges. By using a small region around the detected corner position we can compute the dominant gradient directions within this pattern. These gradient directions are characteristic for the involved edges.

Yin and Balchen [7] describe a method to calculate the dominant gradient directions by statistical analysis of the gradient-directions using the probability density function $(p d f)$. Our approach uses a faster way to estimate the dominant gradient directions, without calculating the $p d f$.

By counting the gradient-directions of all pixels within a small neighborhood of the detected corner-position (and weighting it with the associated gradientmagnitude) and using an interpolation function (see eq.4), a histogram-like function (estimated weighted density function) of the gradient directions can be obtained.

$$
\begin{gathered}
D F(\alpha)=\sum_{i=1}^{n}\left|\operatorname{grad} I\left(x_{i}\right)\right| \phi_{\epsilon}\left(x-x_{i}\right) \\
|\operatorname{grad} I(x)|=\sqrt{\left(\frac{\partial I}{\partial x}\right)^{2}+\left(\frac{\partial I}{\partial y}\right)^{2}} \text { and } \phi_{\epsilon}(x)=\left\{\begin{array}{l}
1 \ldots \text { if }|x| \leq \epsilon \\
0 \ldots \text { else }
\end{array}\right.
\end{gathered}
$$

By varying the parameter $\epsilon$ of the step-function $\phi_{\epsilon}(x)$, the sensitivity of the density function $D F$ can be adapted. Each local maximum of the density function indicates the direction of one involved edge of the detected corner. Figure 1 shows the $7 \times 7$ neighborhood of a corner and the associated bimodal density function.

Experimental Results (see section 3) show that a small $7 \times 7$ neighborhood around the corner position is big enough for a reliable estimation of the gradientdirections. Bigger windows do not deliver significantly more information than a $7 \mathrm{x} 7$ window, but need significantly more computing time. 


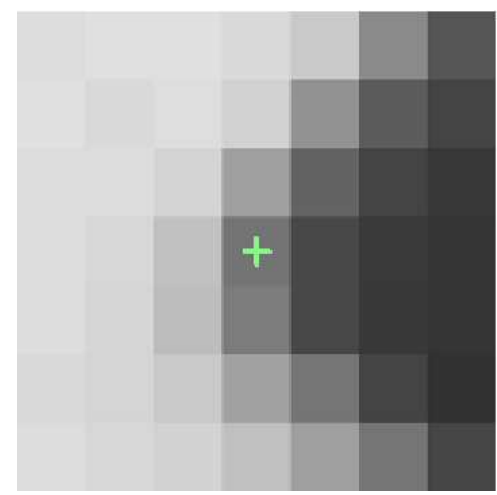

(a)

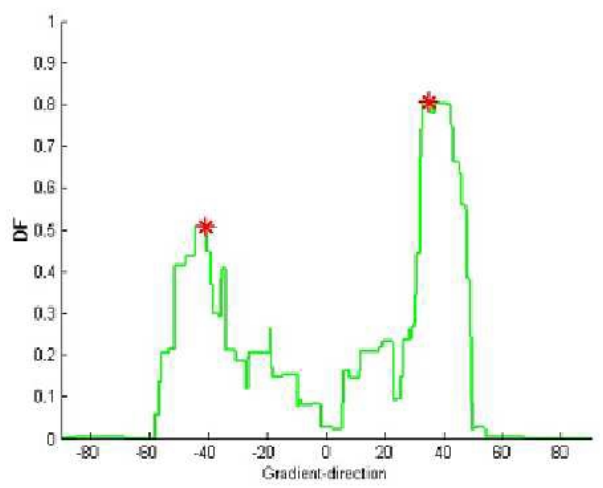

(b)

Fig. 1. (a) $7 x 7$ neighborhood of a detected corner (labeled with a cross). (b) Associated density function with labeled dominant gradient directions

\section{Experimental Results}

To verify the performance of the proposed Similarity Measure, we developed several experimental settings. The following subsections show the sensitivity of the Similarity Measure depending on orientation changes (3.1). In subsection 3.2 we show the redetection performance on real image sequences and compare the matching performance with the tracking performance.

\subsection{Results for Orientation Variation}

The sensitivity for small orientation variations can be shown by a corner image (see figure 2 a) with fixed dihedral angle, which is rotated around the corner position. The step-size between two frames is $1^{\circ}$. Already small changes of the orientation of the corner lead to a significantly lower value of the similarity measure $S M$.

Figure $2 \mathrm{~b}$ shows the associated sensitivity of the Similarity Measure depending on the rotation angle. One can see that the Similarity Measure $S M$ is very sensitive against orientation changes. The two discontinuities of the graph are due to misinterpretations of the gradient density function $D F$ (Wrong maxima detection in DF). 

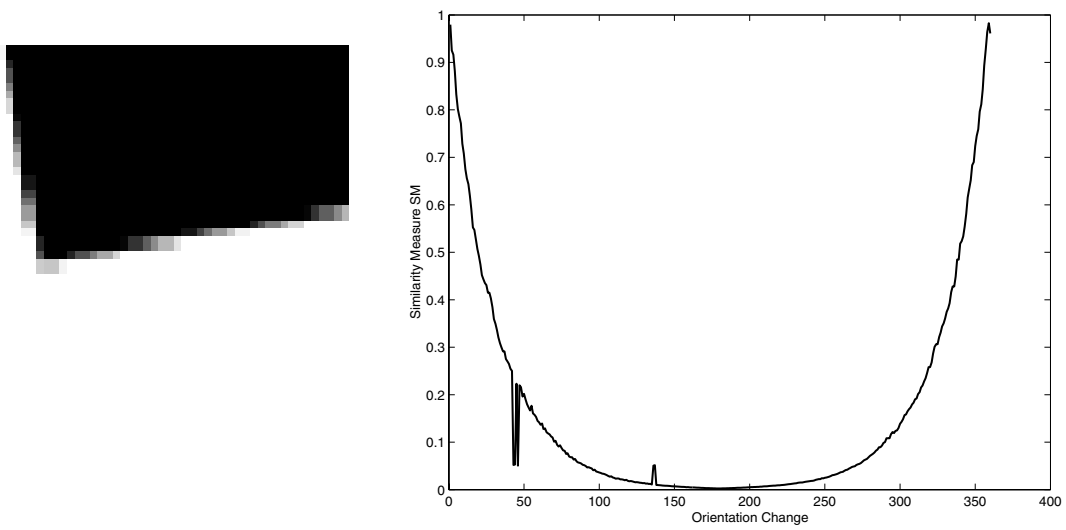

(a)

(b)

Fig. 2. (a) Corner with labeled orientation, which is rotated around the corner position. (b) Associated Similarity Measure, depending on orientation variation.

\subsection{Redetection Results on Real Image Sequences}

Our experimental setup to evaluate the redetection performance of our measure of similarity can be seen in figure 3 a. It consists of several corners with different orientation and different dihedral angle, mounted on an xy-table. Figure $3 \mathrm{~b}$ shows the redetection performance of our similarity measure, depending on the displacement of the corners. The different graphs show on the one hand the redetection performance achieved by using only the initial reference values for the similarity measure (dashed line). On the other hand we use the updated values after each frame (solid line), which is the typical setting for tracking applications.

The results of figure 3 are generated by using the dominant-gradient directions and the cornerness to calculate the similarity measure $S M$.

One can see that the tracking performance, which is the main application of our method is better than the typical matching behavior.

\section{Discussion and Outlook}

This paper presents a new method to calculate a new measure of similarity for corner features, based on additional information provided by a corner, like dominant gradient-direction or cornerness. Without using an explicit metric, we show a simple and fast way to calculate a scaled, easily extendible measure of similarity. The proposed model-based approach can be used for corner redetection of 


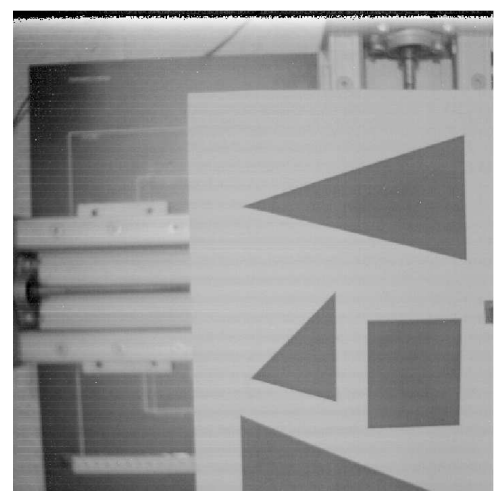

(a)

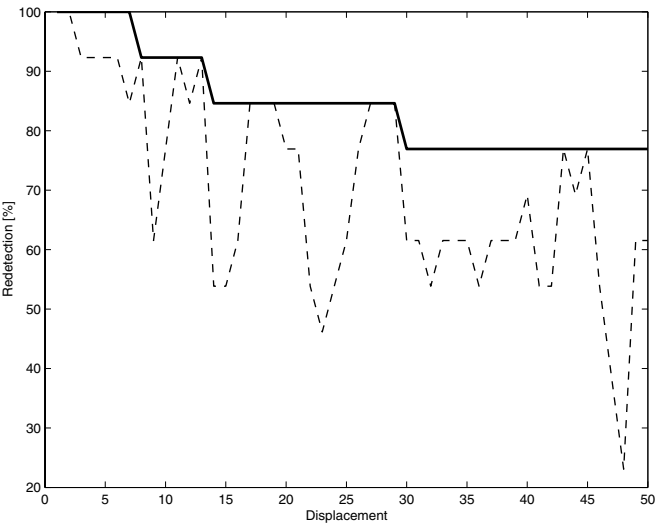

(b)

Fig. 3. (a) Corners with different orientation and dihedral angle mounted on a xy-table to generate various displacements. (b) Associated redetection performance, depending on the application (matching - dashed line vs. tracking - solid line).

well-known corners or as a tool to force the reliability of tracking applications. A high redetection performance can be achieved on real images, especially when using the method for tracking applications.

Currently we can only describe corners, which are intersections of two edges. Future work will focus on arbitrary corners, like T-, Y- or X-junctions with more than two involved edges.

\section{Acknowledgments}

This research was supported by VAMPIRE: Visual Active Memory Processes and Interactive REtrieval (EU-IST Programme IST-2001-34401).

\section{References}

1. Manmohan K. Chandraker, Christoph Stock, and Axel Pinz. Real-time camera pose in a room. In 3rd Intern. Conference on Computer Vision Systems, ICVS 2003, pages 98-110, Graz, Austria, April 2003.

2. C. Harris and M. Stephens. A combined corner and edge detector. In Proc. of the $4^{\text {th }}$ Alvey Vision Conference, pages 189-192, Manchester, 1988.

3. J.Bigun and G. H. Granlund. Optimal orientation detection of linear symmetry. In ICCV, volume 1, pages 433-438, London, June 1987.

4. C. F. Olson and D. P. Huttenlocher. Automatic target recognition by oriented edge pixels. In IEEE Transaction on Image Processing, 6(1), pages 103-113, January 1997. 
5. M. Ribo, H. Ganster, M. Brandner, P. Lang, Ch. Stock, and A. Pinz. Hybrid tracking for outdoor AR applications. IEEE Computer Graphics and Applications Magazine, 22(6):54-63, 2002.

6. Karl Rohr. Recognizing Corners by Fitting Parametric Models. International Journal of Computer Vision, 9(3):213-230, 1992.

7. Yin S. and Balchen J. G. Corner characterization by statistical analysis of gradient directions. In Proc. of Int. Conf. on Image Processing, volume 2, pages 760-763, 1997.

8. Cordelia Schmid and Roger Mohr. Local greyvalue invariants for image retrieval. IEEE Trans. on Pattern Analysis and Machine Intelligence, 19(5):530-535, 1997.

9. P. Smith, D. Sinclair, R. Cipolla, and K. Wood. Effective corner matching. In Proc. 9th British Machine Vision Conference, BMVC 1998, pages 545-556, Southampton, September 1998.

10. Ch. Stock, U. Mühlmann, M. K. Chandraker, and A. Pinz. Subpixel corner detection for tracking applications using CMOS camera technology. In Proc. of 26th Workshop of the Austrian Association for Pattern Recognition(ÖAGM/AAPR), volume 160, pages 191-199, Graz, Austria, September 2002. 